\title{
Soliton-Like Pulse Train Generation Using a Nonlinear Optical Loop Mirror Constructed From Dispersion Decreasing Fiber
}

\author{
Wen-hua Cao and P. K. A. Wai
}

\begin{abstract}
A nonlinear optical loop mirror constructed from dispersion decreasing fiber is used to generate high-quality pulse train from a dual frequency beat signal. Numerical simulations show that, unlike previous techniques based on adiabatic compression in dispersion decreasing fibers which limited the repetition rates to $>50 \mathrm{GHz}$, the proposed method does not use adiabatic compression and can be extended to repetition rates $<20 \mathbf{~ G H z}$ using reasonable fiber lengths.
\end{abstract}

Index Terms-Nonlinear optical loop mirror, nonlinear optics, optical fiber applications, optical pulse compression, optical solitons, optical switching.

\section{INTRODUCTION}

$\mathbf{N}$ ONLINEAR compression of a continuous wave $(\mathrm{CW})$ dual-frequency beat signal is a useful technique for high-repetition rate pulse train generation [1]. Soliton trains at repetition rates of 40-200 GHz have been obtained experimentally using dispersion decreasing fibers (DDF) [1], step-like [2] or comb-like [3] dispersion-profiled fibers, and uniform fibers with distributed amplification [1]. The advantages of this technique include the ability to generate very high-repetition rate pulse trains $(\sim \mathrm{THz})$, very short pulsewidths $(\sim 200$ fs), high output power, and electronic tunability. The main drawback is that impractically long lengths of fiber $(>10 \mathrm{~km})$ are required to extend the technique to repetition rates below $50 \mathrm{GHz}$. Several methods have been used to overcome this difficulty. They include: 1) spectral enrichment of the beat signal in dispersion-shifted fiber (DSF) prior to propagation in DDFs [4]; 2) multisoliton precompression prior to propagation in a DDF [5]; and 3) using nonlinear switching characteristics of a nonlinear amplifying loop mirror (NALM) in conjunction with a dispersion delay line [6]. The decrease in repetition rates by these schemes, however, was not very significant. Although $23-\mathrm{GHz}$ pulse trains were obtained by soliton compression in a $6.45-\mathrm{km}$ constant dispersion fiber [7], the duty cycle of the pulse train was small and the pulse shape deviates significantly from that of a soliton. Generation of soliton trains at 10-20 $\mathrm{Gb} / \mathrm{s}$ remains important because massive wavelength-division multiplexing (WDM) soliton systems with single channel trans-

Manuscript received February 13, 2002; revised May 7, 2002. This work was supported by the Research Grant Council of the Hong Kong Special Administrative Region, China, under Project PolyU5096/98E.

The authors are with Photonics Research Center and Department of Electronic and Information Engineering, The Hong Kong Polytechnic University, Hung Hom, Hong Kong.

Publisher Item Identifier 10.1109/LPT.2002.801840. mission rate of $40 \mathrm{~Gb} / \mathrm{s}$ have yet to overcome impairments such as polarization mode dispersion. High-capacity long-distance WDM soliton systems in the near future will therefore likely be designed with single channel transmission rate of $10-20 \mathrm{~Gb} / \mathrm{s}$ [8].

In this letter, we propose a new scheme which utilizes a nonlinear optical loop mirror constructed from a DDF [9] (hereafter, we call it DD-NOLM). The basic idea is to make use of both the multisoliton pulse compression effect and the switching characteristics of the DD-NOLM. We show numerically that solitonlike pulses at repetition rates less than $20 \mathrm{GHz}$ can be obtained using reasonable loop lengths. We note that although NOLM has been widely used for pulse compression [10], the compressed pulses were not soliton-like. The reason is that the loop length in previous studies (see [10] and the references therein) was chosen such that the clockwise propagating pulse is compressed optimally after traveling around the loop. In fact, this loop length is not optimal for obtaining compressed solitons. We will show that, in order to obtain solitons, the optimum loop length should be longer than that for the clockwise pulses to achieve optimum compression. The DD-NOLM had also been used as an intensity-dependent transmission element in a fiber laser [11]. However, as stated in [11], the mode locking was based on adiabatic pulse compression in loop, thus the loop length would increase exponentially with input pulsewidth (where it was only $1.25 \mathrm{ps).}$ In the proposed scheme, the compression is nonadiabatic which allows one to compress long pulses with short fiber length.

\section{BASIC EQUATIONS}

Pulse propagation in a DDF can be described by [8]

$$
i \frac{\partial u}{\partial \xi}+\frac{1}{2} p(\xi) \frac{\partial^{2} u}{\partial \tau^{2}}+|u|^{2} u=-i \Gamma u
$$

where $u(\xi, \tau)$ is the normalized amplitude and

$$
\begin{aligned}
\xi & =\frac{z\left|\beta_{2}(0)\right|}{t_{0}^{2}}, & \tau & =\frac{t-z / v_{g}}{t_{0}}, \\
p(\xi) & =\left|\frac{\beta_{2}(\xi)}{\beta_{2}(0)}\right|, & \Gamma & =\frac{\alpha}{2} \frac{t_{0}^{2}}{\left|\beta_{2}(0)\right|} .
\end{aligned}
$$

The parameter $\beta_{2}(0)$ is the initial group-velocity dispersion (GVD) coefficient, $v_{g}$ is the group velocity, $\alpha$ is the attenuation constant, $t_{0}$ is a characteristic time, and $p(\xi)$ governs the GVD variation along the DDF. The input is assumed to be [1]

$$
u(0, \tau)=N \sin (\pi \tau / T)
$$




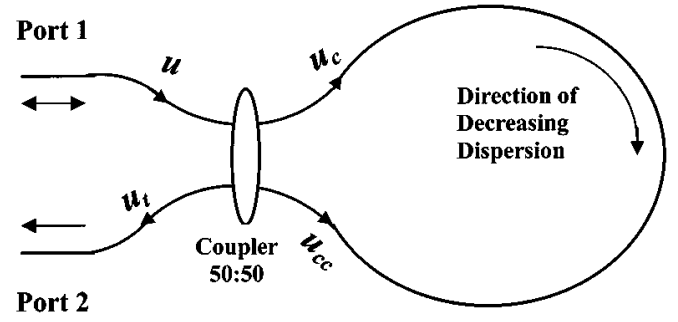

Fig. 1. Schematic of the DD-NOLM.

where $N$ is related to physical parameters by

$$
N^{2}=\frac{\gamma P_{0} t_{0}^{2}}{\left|\beta_{2}(0)\right|}
$$

The parameter $\gamma$ is the nonlinear coefficient and $P_{0}$ is the peak power of the input signal.

\section{RESUlTS AND DisCUSSION}

Fig. 1 shows the configuration of the device. The beat signal is launched into port 1 and the generated pulse train is obtained at port 2. For illustration, we consider an input signal at port 1 in the form of (3) with parameters $N=8$ and $T=2$. The DDF has a linear dispersion profile with an input to output GVD coefficients ratio $G_{\text {eff }}=3$. Using typical values of $\beta_{2}(0)=-20$ $\mathrm{ps}^{2} / \mathrm{km}$ and $\gamma=5 \mathrm{~W}^{-1} \cdot \mathrm{km}^{-1}$ for silica fibers near $1.55 \mu \mathrm{m}$, and assuming a characteristic time of $t_{0}=30 \mathrm{ps}$, we find that the input signal has an average power of $142 \mathrm{~mW}$. Fig. 2(a) and (b) shows the intensity of the pulse train in linear and logarithmic scale, respectively, at a loop length of $7.21 \mathrm{~km}(\xi=0.16)$, where the dashed line in Fig. 2(a) represents the input signal. Fig. 2(c) shows the spectrum while Fig. 2(d) shows the frequency chirp (solid line) and the intensity (dashed line) of a single pulse. The intensities of the pulse shape and spectrum are normalized with respect to the input signal, respectively. Fiber loss is included with $\alpha=0.046 \mathrm{~km}^{-1}(\Gamma \approx 1.04)$. Fig. 2 shows that the generated pulses are pedestal free and their shapes are very close to hyperbolic-secant. The chirp across the main pulse is very small. From its peak intensity and width, the pulse is close to a soliton with a soliton order of 1.26 . The repetition rate of the pulse train is $16.7 \mathrm{GHz}$ with a duty cycle of 8.76 . We note that much longer fiber length must be used if conventional adiabatic techniques [1] were used to obtain the same repetition rate and duty cycle. For example, we consider soliton train generation in a uniform fiber with distributed amplification of $\Gamma=-1$ (as assumed in [1]). The other parameters are $\beta_{2}=-20 \mathrm{ps}^{2} / \mathrm{km}$ and $N=1.1$. The parameter $N$ cannot be chosen too large otherwise the compression will not be adiabatic and the compressed pulses will not be soliton-like. Our results indicate that 57.08 $\mathrm{km}$ of fiber will be needed to obtain results similar to that of Fig. 2.

In Fig. 2, the pulse train is generated from both soliton-effect compression and the switching characteristics of the DD-NOLM. Since the loop is asymmetric, the compression of the counterpropagating signals is different and the two signals acquire different phase shifts when they recombine at the coupler. At the optimum loop length, the switching condition is satisfied for the central peak, but not satisfied for the rest
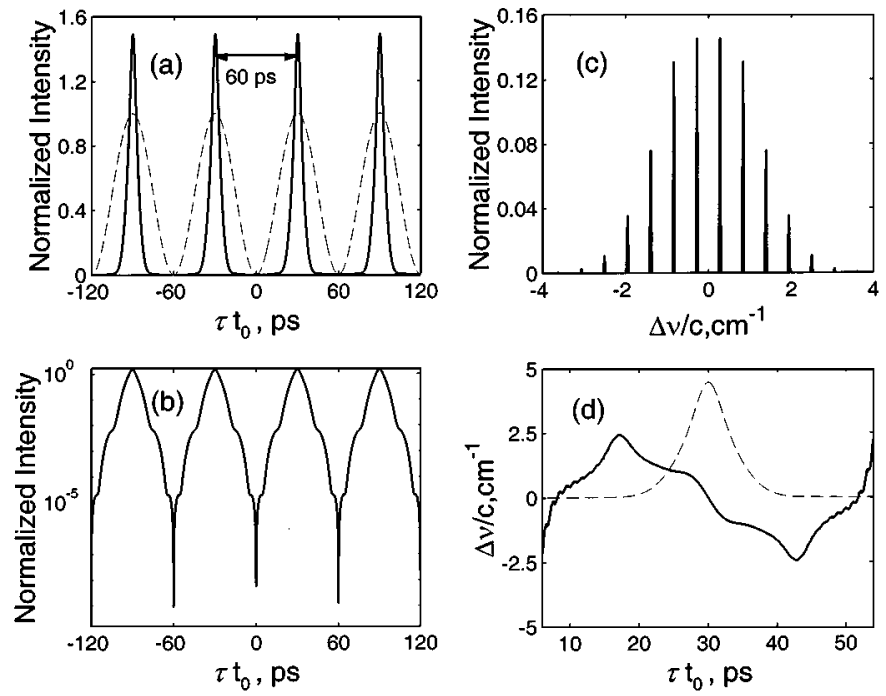

Fig. 2. Temporal intensity of the transmitted pulse train in (a) linear and (b) logarithmic scale. (c) Spectrum of the pulse train and (d) frequency chirp (solid line) and intensity (dashed line) of a single pulse. The dashed line in (a) represents the input signal.

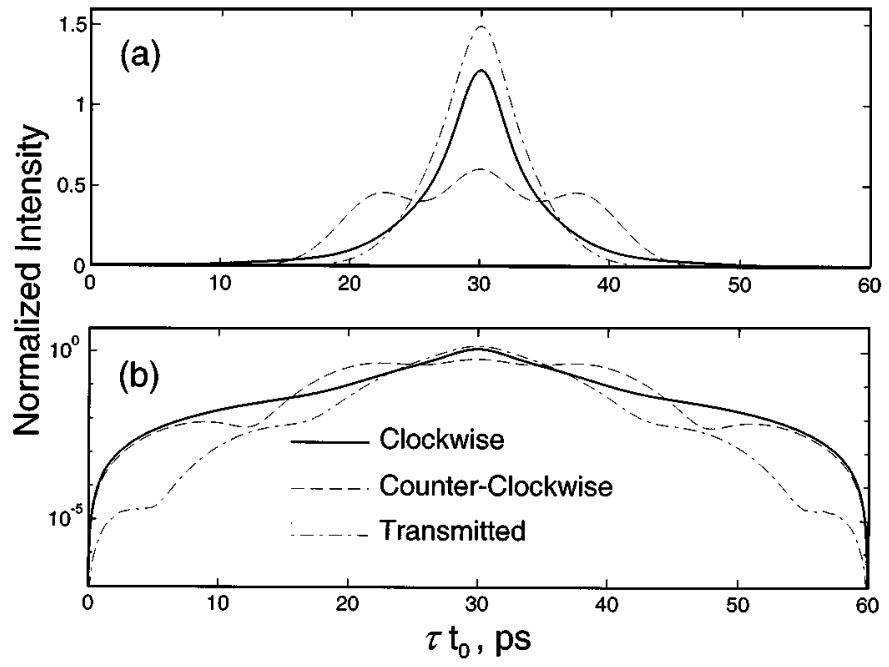

Fig. 3. Clockwise and counterclockwise traveling pulses before recombination: (a) linear scale and (b) logarithmic scale. The transmitted pulse is also shown by the dashed-dotted line.

of the pulse leading to pedestal-free transmitted pulse. One can decrease the optimum loop length for soliton generation or lower the repetition rate while still using reasonable loop length by increasing the input signal power. For example, if we choose $t_{0}=50 \mathrm{ps}, T=2, N=16$ (average power $=205$ $\mathrm{mW}$ ), and $G_{\text {eff }}=2$, then $10-\mathrm{Gb} / \mathrm{s}$ soliton-like pulses with a duty cycle of 15.9 can be obtained with a loop length of 6.99 $\mathrm{km}$. Fig. 3 shows the clockwise and counterclockwise pulses after they travel around the loop but before recombination at the coupler under conditions identical to those of Fig. 2. The transmitted pulse is also shown by the dashed-dotted line. The compression of both the clockwise and counterclockwise pulses are nonadiabatic. The optimum loop length is longer than that for the clockwise pulses to achieve optimum compression.

To demonstrate that the pulses are soliton-like, we couple the pulse train from Fig. 2 into a constant dispersion lossless fiber 


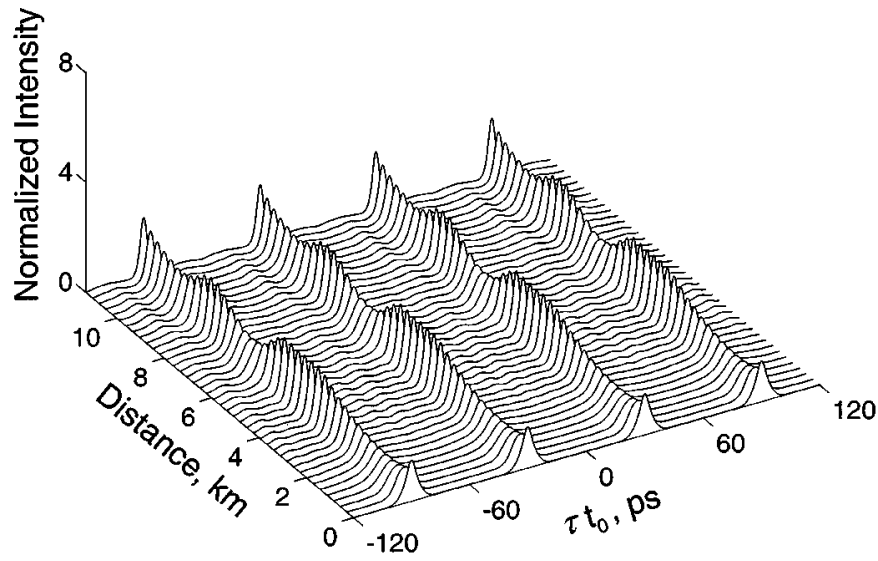

Fig. 4. Propagation of the generated pulse train from Fig. 2(a) in a lossless fiber with constant dispersion.
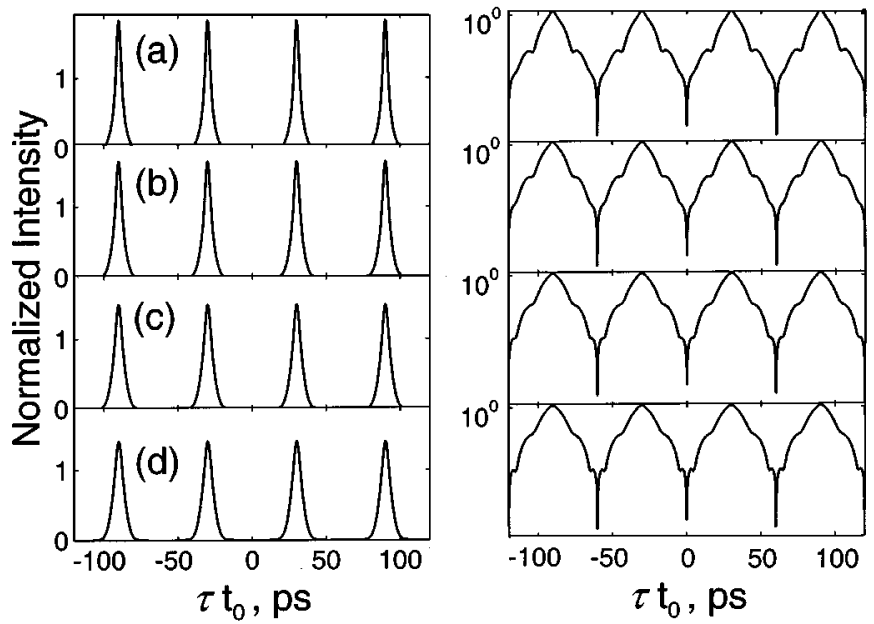

Fig. 5. Pulse shapes of generated pulse trains at loop lengths of: (a) $6.66 \mathrm{~km}$, (b) $6.93 \mathrm{~km}$, (c) $7.2 \mathrm{~km}$, and (d) $7.34 \mathrm{~km}$.

with dispersion coefficient identical to the initial dispersion coefficient $\beta_{2}(0)$ of the DDF. Fig. 4 shows the evolution over a fiber length of $11.3 \mathrm{~km}$ which corresponds to 9.5 soliton periods in terms of the initial pulsewidth. The pulses broaden initially because of the negative chirp [shown in Fig. 2(d)]. They then compressed since their intensities are higher than that of a fundamental soliton. As the pulses continue to propagate, they shed a small part of their energy as dispersive waves and approach fundamental solitons. The dispersive wave is caused by the initial chirp and may increases the Gordon-Haus jitter. One can use fast saturable absorbers such as NOLMs to remove the dispersive wave and reduce the timing jitter. An important feature of the soliton train generated in the proposed scheme is that neighboring solitons have a $\pi$ phase difference which reduces soliton-soliton interaction [1].

Fig. 5 shows the generated soliton shapes at several different loop lengths. In all cases the parameters are identical to those used in Fig. 2 except the loop length (note that the rate of decrease in dispersion is fixed and is identical to that used for Fig. 2). Fig. 5 shows that soliton-like pulses can be achieved over a relatively large range of the loop length. In the range of length studied, the soliton width increases with the loop length because the width of the compressed clockwise pulse increases.

\section{CONCLUSION}

We have proposed a new method to generate soliton pulse trains based on beat-signal compression and switching in a DD-NOLM. The proposed method uses shorter fiber lengths than all previous fiber-based techniques such that repetition rates less than $20 \mathrm{GHz}$ are possible. Although we presented the results for a specific value of $N$ only, we found that the qualitative behavior is the same over a large range of $N$. Therefore, the repetition rate of the pulses can be easily tuned by changing the beating period and input powers.

\section{REFERENCES}

[1] P. V. Mamyshev, S. V. Chernikov, and E. M. Dianov, "Generation of fundamental soliton train for high-bit-rate optical fiber communication lines," IEEE J. Quantum Electron., vol. 27, pp. 2347-2355, Oct. 1991.

[2] S. V. Chernikov, J. R. Taylor, and R. Kashyap, "Experimental demonstration of step-like dispersion profiling in optical fiber for soliton pulse generation and compression," Electron. Lett., vol. 30, no. 5, pp. 433-435, Mar. 1994

[3] — " "Comblike dispersion-profiled fiber for soliton pulse train generation," Opt. Lett., vol. 19, no. 8, pp. 539-541, Apr. 1994.

[4] S. V. Chernikov, D. J. Richardson, R. I. Laming, E. M. Dianov, and D. N. Payne, "70 Gbit/s fiber based source of fundamental solitons at 1550 nm," Electron. Lett., vol. 28, no. 13, pp. 1210-1212, June 1992.

[5] A. V. Shipulin, E. M. Dianov, D. J. Richardson, and D. N. Payne, "40 $\mathrm{GHz}$ soliton train generation through multisoliton pulse propagation in a dispersion varying optical fiber circuit," IEEE Photon. Technol. Lett., vol. 6, pp. 1380-1382, Nov. 1994.

[6] S. V. Chernikov and J. R. Taylor, "Multigigabit/s pulse source based on the switching of an optical beat signal in a nonlinear fiber loop mirror," Electron. Lett., vol. 29, no. 8, pp. 658-660, Apr. 1993.

[7] E. A. Swanson and S. R. Chinn, "23-GHz and 123-GHz soliton pulse generation using two cw lasers and standard single-mode fiber," IEEE Photon. Technol. Lett., vol. 6, pp. 796-798, July 1994.

[8] G. P. Agrawal, Applications of Nonlinear Fiber Optics. San Diego, CA: Academic, 2001.

[9] A. L. Steele and J. P. Hemingway, "Nonlinear optical loop mirror constructed from dispersion decreasing fiber," Opt. Commun., vol. 123, no. 4-6, pp. 487-491, Feb. 1996.

[10] J. Wu, Y. Li, C. Lou, and Y. Gao, "Optimization of pulse compression with an unbalanced nonlinear optical loop mirror," Opt. Commun., vol. 180, no. 1-3, pp. 43-47, June 2000.

[11] A. Boskovic, S. V. Chernikov, and J. R. Taylor, "Femtosecond figure of eight $\mathrm{Yb}$ : Er fiber laser incorporating a dispersion decreasing fiber," Electron. Lett., vol. 31, no. 17, pp. 1446-1448, Apr. 1995. 